\title{
Ret is essential to mediate GDNF's neuroprotective and neuroregenerative effect in a Parkinson disease mouse model
}

\author{
Anja Drinkut ${ }^{1,2}$, Karsten Tillack ${ }^{3,7,8}$, Durga P Meka ${ }^{3,7}$, Jorg B Schulz ${ }^{\star, 1,2,4,9}$, Sebastian Kügler ${ }^{1,5,9}$ and Edgar R Kramer ${ }^{\star, 3,6,9}$
}

Glial cell line-derived neurotrophic factor (GDNF) is a potent survival and regeneration-promoting factor for dopaminergic neurons in cell and animal models of Parkinson disease (PD). GDNF is currently tested in clinical trials on PD patients with so far inconclusive results. The receptor tyrosine kinase Ret is the canonical GDNF receptor, but several alternative GDNF receptors have been proposed, raising the question of which signaling receptor mediates here the beneficial GDNF effects. To address this question we overexpressed GDNF in the striatum of mice deficient for Ret in dopaminergic neurons and subsequently challenged these mice with 1-methyl-4-phenyl-1,2,3,6-tetrahydropyridine (MPTP). Strikingly, in this established PD mouse model, the absence of Ret completely abolished GDNF's neuroprotective and regenerative effect on the midbrain dopaminergic system. This establishes Ret signaling as absolutely required for GDNF's effects to prevent and compensate dopaminergic system degeneration and suggests Ret activation as the primary target of GDNF therapy in PD.

Cell Death and Disease (2016) 7, e2359; doi:10.1038/cddis.2016.263; published online 8 September 2016

Glial cell line-derived neurotrophic factor (GDNF) is the founding member of the four ligands in the GDNF family, which belong to the transforming growth factor- $\beta$ superfamily. ${ }^{1}$ GDNF was characterized as a potent survival factor for many neurons in culture such as dopaminergic, motor, sympathetic, parasympathetic, sensory and enteric neurons. ${ }^{1,2}$ In addition, in dopaminergic neuron cultures GDNF stimulates neuronal differentiation, neurite outgrowth, synapse formation and dopamine release. .,2 $^{1}$

As degeneration of midbrain dopaminergic neurons in the substantia nigra pars compacta (SNpc) represents a major hallmark of Parkinson disease (PD), the most common neurodegenerative movement disorder, GDNF has raised considerable interest as a therapeutic molecule for the treatment of PD. ${ }^{3-5}$ PD affects $>2 \%$ of individuals over the age of 60 years, but no curative treatment is available to date, mainly due to a lack of understanding disease etiology. ${ }^{6-8}$ Preclinical studies in the established 1-methyl-4-phenyl- 1,2,3,6-tetrahydropyridine (MPTP) and 6-hydroxydopamine (6-OHDA) rodent and primate models of PD demonstrated a substantial neuroprotection and regeneration effect by striatal provided GDNF or its close relative neurturin. ${ }^{3,4,9}$ However, clinical phase II trials on PD patients using GDNF or neurturin did so far not convincingly recapitulate their beneficial effects on the dopaminergic system in humans most likely due to technical problems and the selection of advanced PD patients. $^{10-13}$

GDNF signaling is highly complex as this neurotrophic factor can bind to a variety of receptors, thus being able to induce pleiotropic effects. GDNF efficiently binds to the GPIlinked GDNF family receptor $a 1$ (GFR 1 1). ${ }^{1,2}$ It has been shown that the GDNF/GFRa1 complex can activate not only the canonical GDNF receptor Ret, a receptor tyrosine kinase which signals through the sarcoma protein (Src)/rat sarcoma (Ras)/mitogen-activated protein kinase (MAPK), phosphatidylinositol-4,5-bisphosphate 3-kinase (PI3K)/Akt, NF-kB

${ }^{1}$ DFG Research Center Molecular Physiology of the Brain (CMPB), University Medical Center Göttingen, Göttingen, Germany; ${ }^{2}$ Department of Neurodegeneration and Restorative Research, University Medical Center Göttingen, Göttingen, Germany; ${ }^{3}$ Development and Maintenance of the Nervous System, Center for Molecular Neurobiology, University Medical Center Hamburg-Eppendorf, Hamburg, Germany; ${ }^{4}$ Department of Neurology and JARA BRAIN Institute II, RWTH Aachen University and FZ Jülich, Aachen, Germany; ${ }^{5}$ Department of Neurology, University Medical Center Göttingen, Göttingen, Germany and ${ }^{6}$ Department of Applied Physiology, Ulm University, Ulm, Germany

*Corresponding author: JB Schulz, Department of Neurology and JARA BRAIN Institute II, RWTH Aachen University and FZ Jülich, Pauwelsstr. 30, Aachen 52074, Germany; E-mail: jschulz@ukaachen.de

or ER Kramer, Research Group Development and Maintenance of the Nervous System, Centre for Molecular Neurobiology Hamburg (ZMNH), ZMNH, Martinistrasse 52, Hamburg 20246, Germany; and Applied Physiology, Ulm University, Albert-Einstein-Allee 11, 89081 Ulm, Germany. Tel: ++49 $40741055354 /++49731500$ 23879; Fax + +49 407410 55359; E-mail: kramer@zmnh.uni-hamburg.de or edgar.kramer@uni-ulm.de

${ }^{7}$ These authors contributed equally to this work.

${ }^{8}$ Current address: Evotec AG, 22419 Hamburg, Germany.

${ }^{9}$ These authors contributed equally to this work.

Abbreviations: DJ-1, deglycase, oxidative stress sensor and redox-sensitive chaperone and protease; GDNF, Glial cell line-derived neurotrophic factor; GFR $\alpha 1$, GPIlinked GDNF family receptor $\alpha 1$; MAPK, mitogen-activated protein kinase; NF- $\kappa$ B, nuclear factor 'kappa-light-chain-enhancer' of activated B cells, transcription factor family with Rel homology domain; MPTP, 1-methyl-4-phenyl-1,2,3,6-tetrahydropyridine; PD, Parkinson disease; PI3K, phosphatidylinositol-4,5-bisphosphate 3-kinase; PLC $\gamma$, phospholipase $\gamma$ cleaves the phospholipid phosphatidylinositol-4,5-bisphosphate (PIP2) into diacyl glycerol (DAG) and inositol 1,4,5-trisphosphate (IP3); Ras, rat sarcoma; family of small membrane-associated GTPase; SNpc, substantia nigra pars compacta; Src, sarcoma protein; membrane-associated tyrosine kinase with different Src homology (SH) domains characteristic for all nine members of the Src family kinases; $\mathrm{TH}$, tyrosine hydroxylase

Received 28.3.16; revised 29.5.16; accepted 20.6.16; Edited by A Verkhratsky 
(nuclear factor 'kappa-light-chain-enhancer' of activated B cells), JNK (c-Jun N-terminal kinases) and PLC $\gamma$ (phospholipase $\gamma$ ) pathway, but also with other signaling inducing receptors. $^{1,2,4,5,13}$ So far, at least four alternative GDNF receptors have been described which are all expressed in midbrain dopaminergic neurons, $\mathrm{NCAM}^{14,15}$ the integrins $a \mathrm{~V}$ and $\beta \mathrm{I},{ }^{14,16}$ syndecan $3^{17}$ and $\mathrm{N}$-cadherin. ${ }^{18}$ Interestingly, Ret is not essential during pre- and postnatal development of the mouse dopaminergic system, ${ }^{19-23}$ but specifically required for the maintenance of SNpc dopaminergic neurons and their striatal innervation in aged mice. ${ }^{23-25}$ In contrast, GDNF seems most likely under physiological conditions to be dispensable during development and maintenance of midbrain dopaminergic neurons in mice, although conflicting results exist. ${ }^{26-28}$ Thus, Ret might be activated by a GDNFindependent mechanism to stimulate SNpc dopaminergic neuron survival. In addition, the in vivo function of the alternative GDNF receptors in the dopaminergic system under physiological and pathophysiological conditions, like PD, and their dependence on GDNF has not yet been addressed in detail. This raised the important question which GDNF receptor might be required to mediate GDNF's reported neuroprotective and regenerative effect in the dopaminergic system in PD animal models and potentially in PD patients. 5,29

Previously, we showed in dopaminergic neuron-specific Ret knockout mice that Ret receptor loss does not result in a higher vulnerability of midbrain dopaminergic neurons against MPTP but to less resprouting of left over dopaminergic neuron axons in the striatum after MPTP intoxication. ${ }^{30}$ In adult mice endogenous GDNF levels are rather low. ${ }^{26,31}$ Therefore, we could not rule out in that study the possibility, that higher levels of GDNF-as also used in the clinical GDNF trials in PD patients-might have neuroprotective and regenerating effects even in the absence of the Ret receptor. Here we addressed now this question by viral overexpression of GDNF in MPTP-treated mice lacking expression of Ret again specifically in dopaminergic neurons. ${ }^{23,30}$ We found that in the absence of Ret in dopaminergic neurons even a substantial overexpression of GDNF in the striatum does not have a neuroprotective and regenerative effect. Thus, despite the expression of alternative GDNF receptors on midbrain dopaminergic neurons, the presence of the canonical GDNF receptor Ret seems to be mandatory for mediating GDNF's beneficial survival and axonal resprouting effect in these neurons.

\section{Results}

To investigate the Ret receptor function in mediating the neuroprotective and neuroregenerative effects of GDNF we generated mice with a disrupted Ret gene specifically in dopaminergic neurons (DAT-Ret $\left.{ }^{\mid x / 1 x}\right)^{23}$ by crossing mice carrying the floxed allele of Ret $\left(\operatorname{Ret}^{\mid x / 1 x}\right)^{32}$ with dopamine transporter (DAT) promoter-driven Cre mice (DAT-Cre). ${ }^{33} \mathrm{We}$ overexpressed GDNF in these mice by stereotactic injection of a recombinant adeno-associated virus serotype 5 (AAV5) encoding the mouse GDNF cDNA under the control of the glial fibrillary acidic protein (GFAP) promoter (AAV5-GDNF) unilaterally in the striatum of the left mouse brain hemisphere (Figure $1 \mathrm{~g}$ and $\mathrm{h}$ ). ${ }^{34}$ The contralateral hemisphere of the same animal served as an internal control as we found that this expression mode restricted the delivery of GDNF to only the vector-injected hemisphere (Figure 1g). ${ }^{34}$ Besides injecting a control virus encoding the enhanced green fluorescent protein under the GFAP promoter (AAV5-EGFP) in some mice confirming astrocyte-specific transgene expression, we also unilaterally sham-injected some mice (Figure 1a-f). Two weeks after injection of AAV5-GDNF we determined by immunohistochemical (IHC) and enzyme-linked immunoabsorbent assay (ELISA) the striatal GDNF levels to be $87961 \mathrm{pg} / \mathrm{mg}$ tissue and the nigral GDNF levels of $1097 \mathrm{pg} /$ $\mathrm{mg}$ tissue, whereas in AAV5-EGFP-injected animals striatal GDNF levels were 40 and $18 \mathrm{pg} / \mathrm{mg}$ tissues in the nigra. Thus, we achieved with the AAV5-GDNF vector an around 2200-fold and 61 -fold overexpression of GDNF in the striatum and in the SNpc compared with AAV5-EGFP-injected mice, respectively (Figure 1g; Supplementary Figure 1). GDNF is spreading over the whole brain hemisphere sterotactically injected including the striatum and cortical regions (Supplementary Figure 1). The leakage of GDNF to the contralateral side is only threefold in the striatum and 12-fold in the substantia nigra (Figure 1g).

In our paradigm 1 week after virus injection the mice were challenged with a systemic subchronic MPTP treatment leading to $40-60 \%$ of dopaminergic system lesion or were treated with saline $(\mathrm{NaCl})$ as a control (Figure 1h). ${ }^{30}$ The nigrostriatal dopaminergic system of these mice was analyzed 2 weeks later to monitor the neuroprotective effect of GDNF and three months after the MPTP treatment to investigate the neuroregenerative potential of GDNF (Figure 1h). ${ }^{30}$

First, we stereologically quantified the number of tyrosine hydroxylase (TH)-positive dopaminergic neurons of the SNpc in the mouse brains 2 weeks after MPTP treatment. In all three genotypes (DAT-Cre, Ret $^{1 \times / 1 x}$ and DAT-Ret ${ }^{\mid x / 1 x}$ ) MPTP reduced the number of dopaminergic neurons by $46 \%$ (3000-4000 cells per brain half) compared with saline ( $\mathrm{NaCl}$ )-treated mice (Figure 2a). Injection of AAV5-EGFP did not reduce MPTPinduced cell death in any genotype (Figure 2a). As expected, GDNF was neuroprotective in the AAV5-GDNF-treated brain hemisphere of control mice (DAT-Cre and Ret ${ }^{\mathrm{I} / \mathrm{Ix}}$ ) and MPTP induced $<20 \%$ loss of dopaminergic neurons (Figure 2a). In contrast, GDNF expression conferred no neuroprotective effect against MPTP in Ret-deficient mice (DAT-Ret ${ }^{\mid \mathrm{x} / \mathrm{x}}$ ). Thus, in the absence of Ret, even substantial amounts of GDNF did not protect dopaminergic neurons from MPTP toxicity. The same cell numbers were also observed in mice analyzed 3 months after MPTP treatment confirming the long-term neuroprotective effect of GDNF/Ret on dopaminergic neurons and also the general lack of dopaminergic cell regeneration after MPTP treatment independent of GDNF overexpression or not (Figure 2b). We did not observe a significant motor impairment in the MPTP-treated mice compared with the $\mathrm{NaCl}$-treated mice using the open field, Rotarod and tight rope test, and could therefore not follow GDNF's beneficial effect on the behavior (data not shown). This is most likely due to $<50 \%$ dopaminergic cell loss with the MPTP treatment (Figure 2).

Next, we investigated if GDNF's dopaminergic innervation protective function after MPTP treatment depends on the Ret receptor. Therefore, we assessed $\mathrm{TH}$ - and DAT- positive dopaminergic fibers in the striatum of the three genotypes 2 weeks and 3 months after MPTP intoxication (Figure 3; 

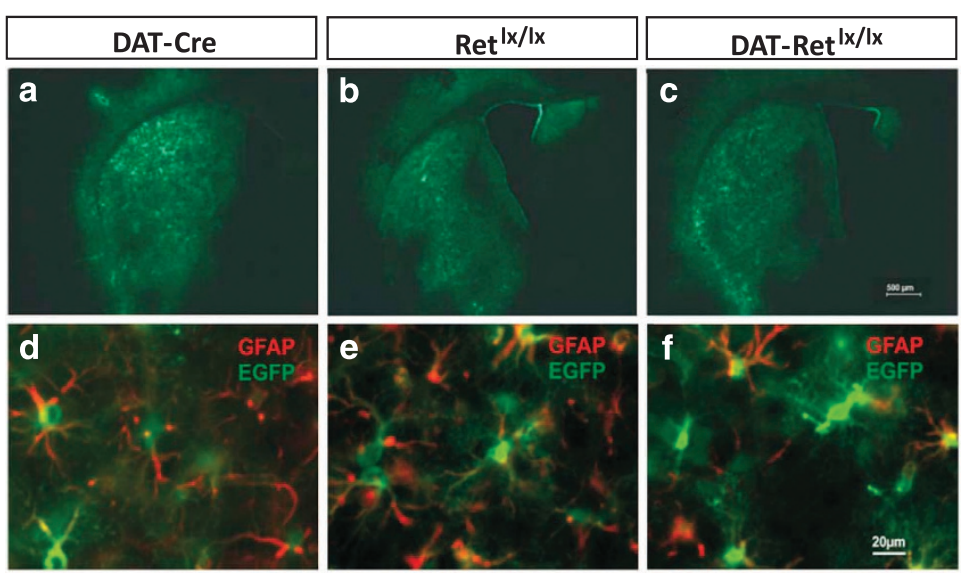

h
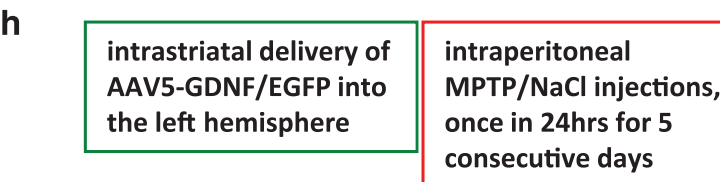
brain tissue analysis for substantia nigra dopaminergic cell counts, dopaminergic innervation of the striatum, and levels of dopamine metabolites in the striatum

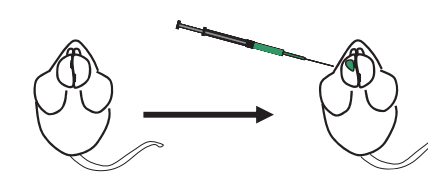

consecutive days

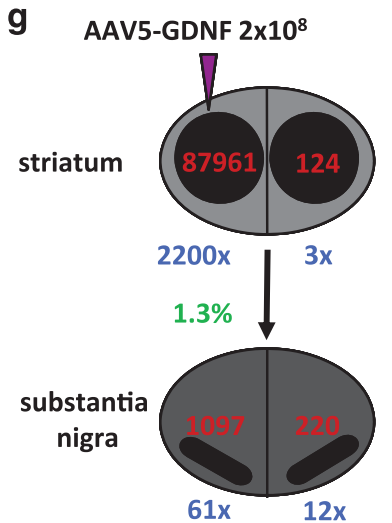

DAT-Ret ${ }^{\text {Ix/lx }}$
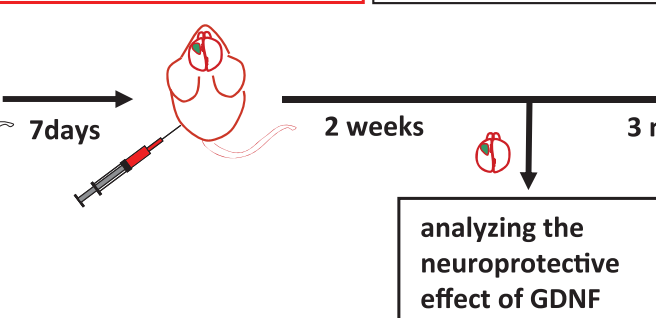

3 months

controls

Figure 1 Tools and paradigm to address Ret function in mediating GDNF neuroprotective and regenerative effect in the MPTP mouse model of PD. (a-f) Coronal striatum sections are shown for the three mouse genotypes (DAT-Cre, Ret ${ }^{\mid x / x}$ and DAT-Ret ${ }^{\mid x / x}$ ) which were transduced with an adeno-associated virus of the serotype 5 encoding EGFP under the GFAP promoter to target astrocytes (AAV5-EGFP). (a-c) EGFP expression in the striatum of the left brain hemisphere at low resolution (scale bar, $500 \mu \mathrm{m}$ ). (d-f) EGFP expression in green co-localizes in astrocytes immunohistochemically stained in red with antibodies against GFAP (scale bar, $20 \mu \mathrm{m}$ ). (g) Schema shows absolute values of GDNF measured by ELISA in pg/mg tissue (red numbers) and fold of GDNF overexpression (blue numbers) of AAV5-GDNF injected mice in comparison to AAV5-EGFP injected wild-type animals with $40 \mathrm{pg} \mathrm{GDNF} / \mathrm{mg}$ tissue in the striatum and $18 \mathrm{pg} \mathrm{GDNF} / \mathrm{mg}$ tissue in the nigra. Only $1.3 \%$ of the GDNF expressed in the infected striatum is retrograde transported into the ipsilateral substantia nigra (green number). (h) Schema illustrating the experimental paradigm used

Supplementary Figures 2-5). Two weeks after MPTP treatment the striatal dopaminergic innervation was reduced by almost $50 \%$ in all three genotypes compared with $\mathrm{NaCl}-$ treated mice (Figure 3a). Injection of AAV5-EGFP had no influence on the MPTP-induced striatal dopaminergic axon loss (Figure 3a). Although the AAV5-GDNF-treated brain hemisphere of control mice treated with MPTP showed almost no degeneration of the striatal dopaminergic innervation, no protection was detectable in DAT-Ret ${ }^{\mid x / 1 x}$ mice (Figure $3 b$ ). Besides quantifying the 3,3-Diaminobenzidin (DAB) staining of the TH-positive fibers in the striatum (Figure 3), which does not allow to distinguish between alterations in $\mathrm{TH}$ protein and alterations in the amount of TH-positive fibers, we also performed a fluorescent TH staining and quantified precisely the relative density of dopaminergic fibers in the dorsal and ventral striatum using TH and DAT as markers (Supplementary Figures 2-5). These experiments support the DAB data.

To investigate the extent to which the residual dopaminergic fibers in the striatum remained functional, we quantified the total concentration of dopamine and its metabolites 3,4-dihydroxyphenylacetic acid (DOPAC) and homovanillic acid (HVA) in the striatum two weeks after MPTP treatment
(Figure 4a; Supplementary Figure 4). Consistent with the dopaminergic fiber density also dopamine, DOPAC and HVA levels were only protected in the GDNF expressing hemisphere of DAT-Cre and Ret ${ }^{1 / / x}$ mice but not in DAT-Ret ${ }^{\mid x / x}$ mice (Figure $4 \mathrm{a}$ ). This shows that the protective function of exogenous GDNF on dopaminergic cell bodies and axons in the MPTP model depends on the presence of the Ret receptor in dopaminergic neurons.

Finally, we assessed GDNFs regeneration function at 3 months after MPTP in the three genotypes by quantifying $\mathrm{TH}-$ and DAT-positive fibers in the striatum (Figure 3c; Supplementary Figures 5 and 6). As observed previously, ${ }^{30}$ resprouting of the residual dopaminergic fibers after partial denervation by MPTP strictly depends on the presence of the Ret receptor and is already induced by endogenous levels of GDNF (about $0.04 \mathrm{ng} / \mathrm{mg}$ tissue; Figure $3 \mathrm{c}$ ). Thus, substantial regeneration of dopaminergic fibers to $70-80 \%$ of saline injected control mice was detected in MPTP-treated DAT-Cre and $\operatorname{Ret}^{\mathrm{Ix} / \mathrm{x}}$ mice in the non-AAV-injected control brain site as well as in the AAV5-EGFP injected hemispheres, but not in Ret-deficient mice (Figure 3c). A significant additional regenerating effect of up to $90-100 \%$ of $\mathrm{NaCl}$-treated control 


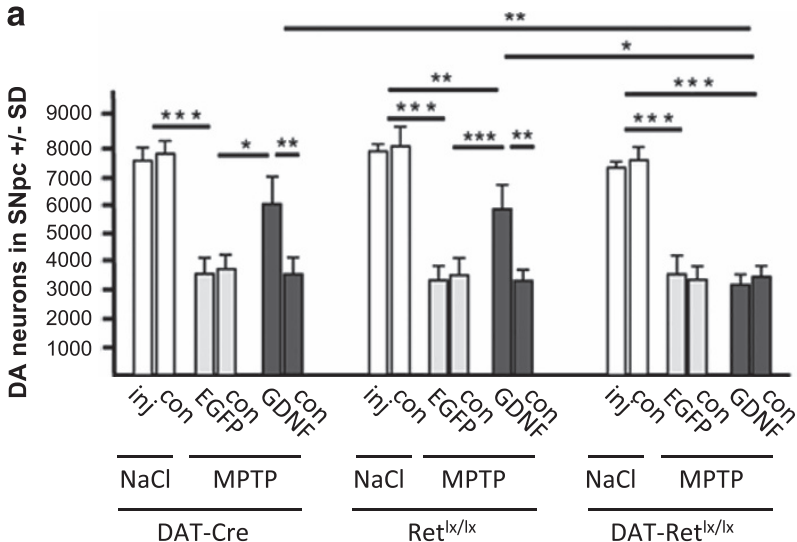

b

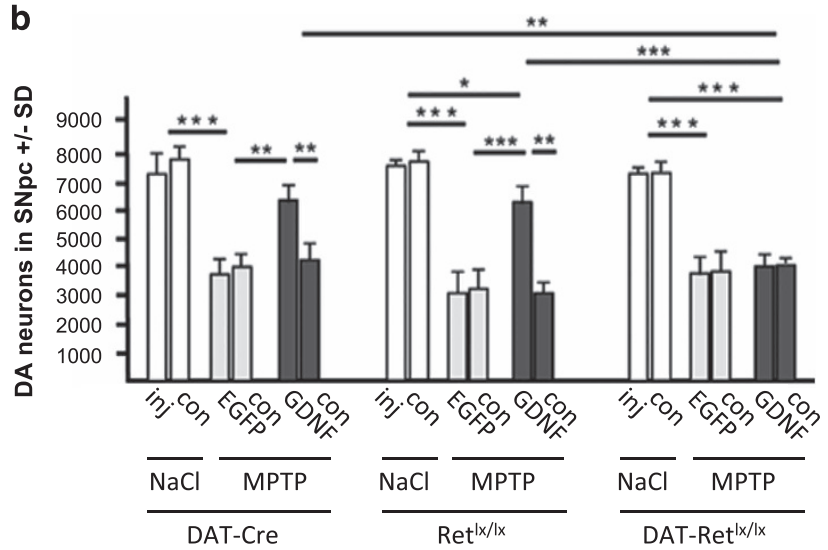

Figure 2 Stereological quantification of nigral dopaminergic neurons. Numbers of TH and Nissl-positive nigral neurons are shown for the three genotypes (Dat-Cre, Ret ${ }^{\mid x / x}$ and DAT-Ret $\left.{ }^{\mid \mathrm{l} / \mathrm{l} x}\right) 2$ weeks post MPTP treatment (a) and 3 months after MPTP treatment (b) and control mice which instead received saline ( $\left.\mathrm{NaCl}\right)(\mathbf{a}$ and $\mathbf{b})$. For each mouse the numbers on the side with mock injection (inj), AAV5-EGFP injection (EGFP) or AAV5-GDNF (GDNF) and on the non-injected contralateral side (con) are shown ( $n=6$ animals per group)

mice was detectable in the ipsilateral of AAV5-GDNF injected and MPTP-treated DAT-Cre and Ret ${ }^{1 / 1 / x}$ mice (Figure $3 c$ ). But even after high- and long-term overexpression of GDNF no regeneration of dopaminergic fibers was detectable in the striatum of DAT-Ret ${ }^{\mid x / x}$ mice (Figure 3c). The quantification of fluorescently labeled fibers reveals besides GDNF's neuroprotective effect also the significant resprouting of $\mathrm{TH}$-positive fibers in the two control groups between 2 weeks and 3 months from 18 to $37 \%$ on the AAV5-GFP injected side of MPTP treated mice and 43 to $68 \%$ on the AAV5-GDNF injected side of MPTP treated mice compared with sham-injected mice (Supplementary Figures 2 and 4). We conclude that resprouting of dopaminergic axons after MPTP intoxication even in the presence of exogenous GDNF strictly depends on the presence of the Ret receptor. We also determined striatal levels of dopamine and its metabolites DOPAC and HVA by HPLC to rule out that GDNF might not influence the physiologically important neurotransmitter levels even in the absence of dopaminergic fiber regeneration in the Retdeficient mice (Figure 4b; Supplementary Figure 4). However, in contrast to control mice where dopamine levels increased corresponding to the degree of regeneration no recovery of the dopamine levels took place in Ret-deficient mice demonstrating that also no functional recovery of the dopaminergic system is possible in the absence of Ret (Figure 4b).

To find out if our AAV5 vectors have a general effect on the brain physiology we also analyzed the glia response in our mice (Supplementary Figure 7). Although we did not find alterations in the number of microglia quantified with antibodies against ionized binding calcium adapter molecule (lba)-1 in the SN and striatum, they led to a low, but persistent, increase in GFAP positive astrocyctes in the striatum 3 months after the MPTP treatment in addition to the previously described transient increase of astrocytes at two weeks after the MPTP treatment ${ }^{30}$ (Supplementary Figure 7). This mild gliosis seem not to be influenced by GDNF expression and seems also not to interfere with the observed beneficial effect of GDNF/Ret signaling on dopaminergic neuron regeneration.

\section{Discussion}

We described in Kowsky et al. ${ }^{30}$ already that the absence of Ret does not decrease MPTP vulnerability leading to the same MPTP induced cell loss but prevents subsequent regeneration of dopaminergic fibers most likely mediated by endogenous GDNF. Here, in this manuscript, we overexpress now in addition GDNF before the MPTP treatment (Figure 1). This allows us to show in Ret expressing mice 2 weeks after MPTP treatment the neuroprotective effect of GDNF on the dopaminergic cell bodies and fibers in the striatum and additionally 3 months after MPTP treatment a more pronounced stimulatory effect of GDNF on regenerating dopaminergic fibers in the striatum (Figure 3; Supplementary Figures 2-5). Thus, indeed, we could show different effects of GDNF/Ret signaling on the midbrain dopaminergic cell body and the striatal dopaminergic innervation. The dopaminergic cell bodies require Ret for exogenous GDNF-induced protection from MPTP toxicity, although enhanced GDNF/Ret signaling cannot induce cell proliferation (Figure 2). In addition, Ret is required in the dopaminergic axons to mediate there exogenous GDNF's protective and regenerative effect (Figure 3; Supplementary Figures 2-5). This exogenous GDNF protects and also allows to partially recover the dopamine levels in the presence of Ret (Figure 4) suggesting for exogenous GDNF not only a beneficial effect on the fiber histology but also on the physiology. As the absence of Ret abolished already completely GDNF's neuroprotective function there was no need to apply GDNF after the MPTP induced degeneration to investigate only GDNF's regenerative function. Although the MPTP model of PD does not mimic all aspects of idiopathic PD, ${ }^{35,36}$ it is a robust lesion model that allowed us to assess the requirement of Ret-mediated GDNF signaling. In our previous work we already established that Ret loss does not alter MPTP metabolism. ${ }^{30}$

Our result, that GDNF can provide its neuroprotective and neuroregenerative function on midbrain dopaminergic neurons only in the presence of the receptor tyrosine kinase Ret, has practical implications for therapeutic strategies to treat $P D$ with GDNF and neurturin. ${ }^{3,7,11,12,13}$ It suggests that the main 
a

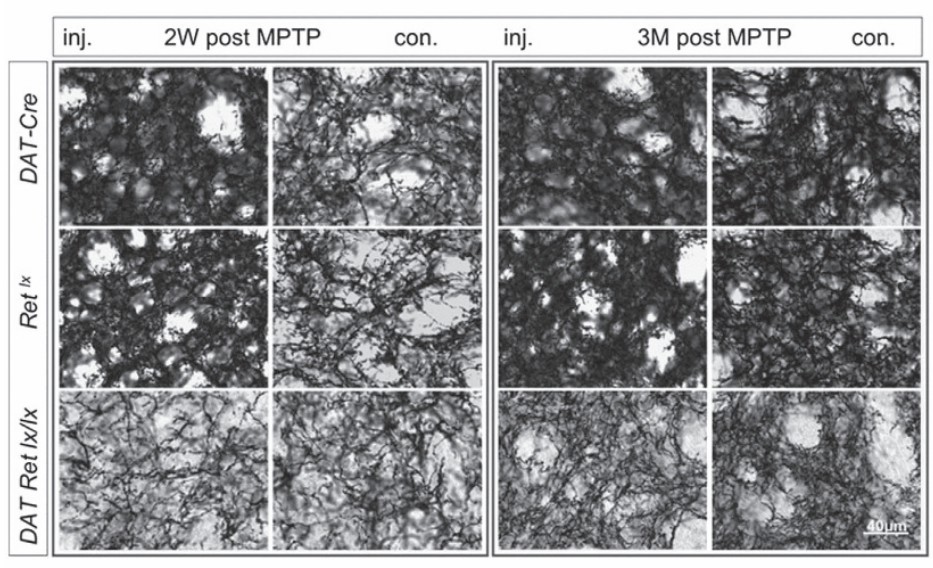

b

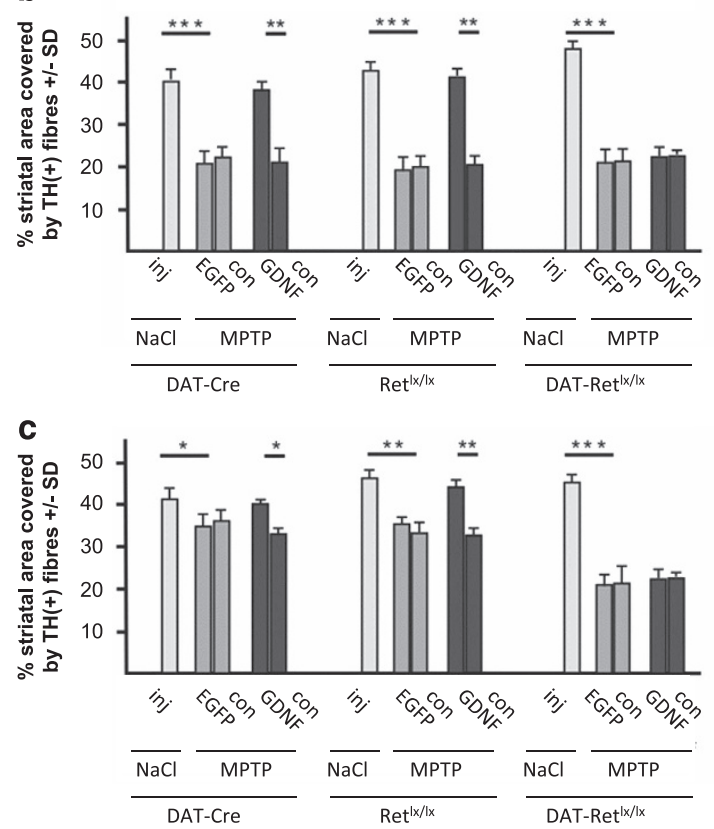

Figure 3 Quantification of striatal dopaminergic fiber density. TH immunoreactivity of striatal tissue sections is shown in a for DAT-Cre mice (upper panel), Ret ${ }^{\mid \mathrm{x} / \mathrm{x}}$ mice (middle panel) and DAT-Ret ${ }^{\mid x / x}$ mice (lower panel) at 2 weeks after MPTP treatment (first two columns) and 3 months after MPTP treatment (last two columns), for the injected hemispheres (inj., columns 1 and 3) and the contralateral hemispheres (con, columns 2 and 4). The quantification of striatal TH fiber density is presented in (b) for 2 weeks, and in c for 3 months after MPTP application. Scale bar, $40 \mu \mathrm{m}$ in (a) ( $n=6$ animals per group)

goal of clinical trials using GDNF and related substances should be to activate the Ret receptor. In our experiments even striatal GDNF levels 2000-fold above endogenous levels for $>3$ months were not sufficient to reveal any Ret-independent neuroprotective or regenerative effect of GDNF in the MPTP mouse model of PD. As the Ret receptor is only expressed in the dopaminergic system in dopaminergic neurons of the midbrain and GDNF expression was not able to suppress the mild but long-term gliosis in the SNpc, this supports the idea that GDNF stimulates directly intrinsic survival and resprouting pathways in dopaminergic neurons and additional effect on glia cells and other neurons seem less important. Thus, GDNF can only function efficiently when enough dopaminergic neurons are still around which express the Ret receptor., ${ }^{5,29}$ Late stage PD patients with too few dopaminergic cells and strongly reduced striatal dopaminergic innervation will most likely not be able to respond efficiently to GDNF and should be excluded from the clinical GDNF trials. A PD therapy based on stimulating Ret signaling in dopaminergic neurons of the SN should be most effective in the early phase of the disease when there are still many dopaminergic neurons present on which Ret can mediate survival of dopaminergic neurons, protection from toxic insults, and resprouting of fibers in the striatum. ${ }^{3,7,11,12,13}$ Neurturin binds with high affinity on GFR $a 2,{ }^{1}$ which is expressed in the ventral midbrain but not on midbrain dopaminergic neurons. ${ }^{37-39}$ This suggests that neurturin might stimulate Ret on dopaminergic neurons by low affinity binding to GFRa1 or supports dopaminergic neurons in a non-cell-autonomous manner. ${ }^{39}$
Interestingly, endogenous GDNF has been shown to be expressed in the adult striatum only in fast spiking, parvalbumin-positive GABAergic interneurons-representing $0.7 \%$ of all striatal neurons-and to a lower extent in some somatostatinergic and cholinergic interneurons, but not in medium spiny neurons. ${ }^{13,31,40}$ As GDNF cannot pass the blood-brain barrier (BBB), exogenous GDNF must be provided for the clinical trials by specific routes. GDNF protein can be intrastriatal or intranasal infused, modified or packaged GDNF that can pass the BBB might be supplied systemically, GDNF encoding DNA constructs might be provided by viral vectors, or GDNF expressing cells might be transplanted into the striatum. ${ }^{3,7,11,12,13,41}$ Previously we compared astrocytic (human GFAP promoter) and neuronal (human synapsin 1 promoter) expression levels of GDNF with the AAV5 serotype vector and found similar GDNF expression levels in the injected side but less spreading to the contralateral side using the GFAP promoter. ${ }^{34}$ It has already been previously shown that AAV serotype 5 allows wide spreading of the virus in the striatum and efficient targeting of astrocytes. ${ }^{34,42}$ The GFAP promoter led to high- and long-term GDNF expression in astrocytes without adverse effects as also shown previously in adeno- and lentivirus vectors. ${ }^{34,43,44}$ This is in contrast to high expression of GDNF in striatal and nigral neurons which can lead to negative side effects, such as downregulation of $\mathrm{TH}$ protein levels. ${ }^{45-48}$ If in clinical trials the amount of bioactive GDNF in the striatum is limiting, the here used AAV5-GDNF serotype 5 vector with the GFAP promoter seems to be an efficient and safe alternative to provide GDNF ${ }^{34}$ Further experiments are needed to clarify if the mild striatal gliosis 
a

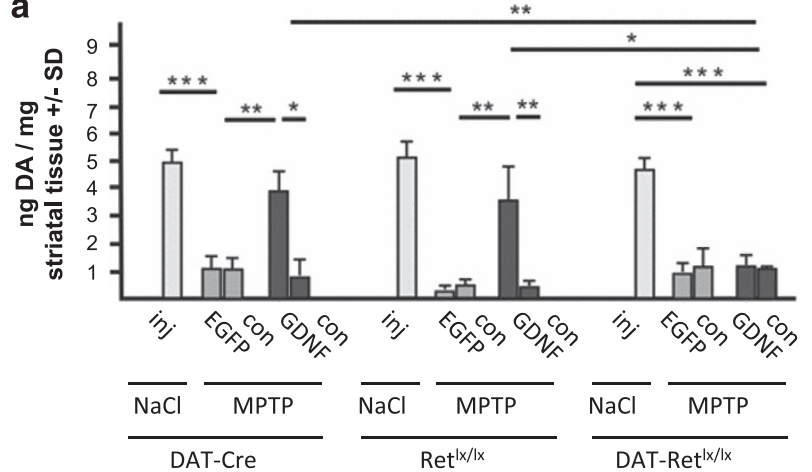

b

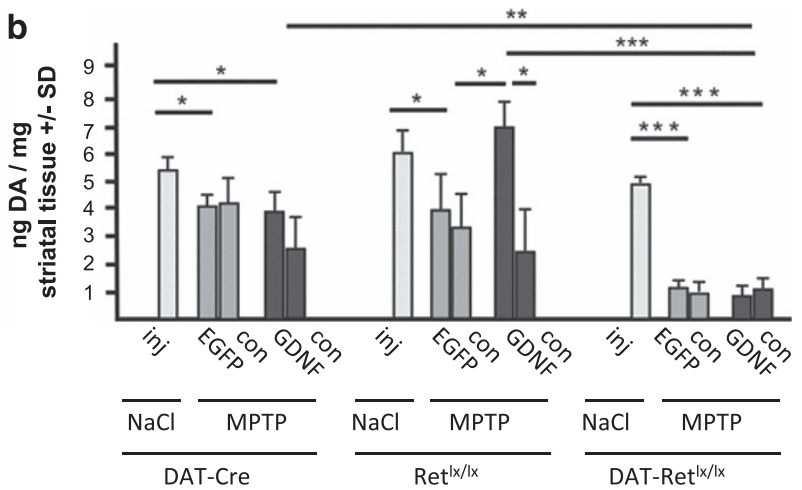

Figure 4 Quantification of striatal dopamine levels. Striatal dopamine levels assessed by HPLC are shown for control animals (NaCl), for animal receiving MPTP and AAV5EGFP (MPTP+EGFP), and for animals receiving MPTP and AAV5-GDNF (MPTP+GDNF) for the vector injected (inj) and the contralateral hemisphere (con). Data are shown from the three genotypes (Dat-Cre, Ret ${ }^{\mid x / x}$ mice and DAT-Ret ${ }^{\mid x / x}$ ) at 2 weeks (a) and at 3 months post MPTP treatment $(\mathbf{b})(n=6$ animals per group)

found in our mice is also observed in humans and if this results in negative side effects.

Previous work has already shown that GDNF's neuroprotective function requires not only Ras-MAPK activation but also PI3K-Akt signaling. ${ }^{5,30,49-51}$ It is also well established that the Ret receptor it essential for maintaining aged SNpc dopaminergic neurons and seems to crosstalk for this maintenance function with proteins such as the chaperon DJ-1, PINK-1 (PTEN-induced putative kinase 1) and parkin, an E3 ubiquitin-protein ligase, for which mutated forms were found to lead to PD. ${ }^{5,24,25,29,52,53}$ Ret and DJ-1 (deglycase) trigger dopaminergic cell survival in mice by activating together the Ras/MAPK pathway. ${ }^{24}$ Ret crosstalks with PINK-1 and parkin to ensure mitochondrial morphological and physiological integrity in dopaminergic neurons..$^{25,52,53}$ Ret and parkin are not only important for survival of aged dopaminergic cell body in the SNpc but also for the maintenance of the striatal dopaminergic innervation. ${ }^{5,25,29,53}$ Therefore, we suspect that these signaling events downstream of the Ret receptor play also an important role in mediating GDNF's neuroprotective and regenerative function in the dopaminergic system.

Although we have shown here the requirement for Ret for mediating GDNF's neuroprotective and regenerative effect in midbrain dopaminergic neurons, this does not rule out an additional function for alternative GDNF receptors in this context and also for mediating survival during development and maintenance of these neurons. The recent finding, that GDNF might be dispensable for the development and maintenance of the dopaminergic system in mice $e^{26,27,28}$ supports the idea, that alternative GDNF receptors might even function without the need to bind GDNF as important signaling recognition or cell adhesion molecules in dopaminergic neurons. Further studies are needed to clarify the physiological function of alternative GDNF receptors and GDNF itself in mice and man.

Small molecules that specifically activate Ret and can cross the BBB would be ideal and seem to be an achievable goal as small non-peptidyl quinol based GFRa1 agonists have already been developed. ${ }^{54,55}$ Such substances would not only simplify the application procedure but would presumably also decrease the chance of unwanted side effects that might result from interaction with alternative GDNF receptors. If the Ret protein on dopaminergic neurons is limiting, Ret might also be provided by a viral vector approach targeting directly dopaminergic neurons. ${ }^{10}$ Such scenario has been created by high viral overexpression of $a$-synuclein in rats leading to downregulation of the orphan nuclear receptor Nurr-1 and its downstream target Ret. ${ }^{56}$ $a$-Synuclein is aggregating in Lewy bodies and neurites frequently observed in PD patients and gene amplifications and mutations of $a$-synuclein are found in some familiar forms of PD. ${ }^{8,57}$ However, so far $\mathrm{no}^{58}$ or only mild ${ }^{56}$ reduction of Ret mRNA and protein levels have been found in PD patients, so that more investigations are needed to clarify this issue.

Taken together, our data support the ongoing clinical phase I trials on PD patients testing AAV-encoded GDNF and phase II trials using recombinant GDNF protei ${ }^{12}$ and emphasize the central role of Ret in mediating GDNF's survival and regeneration signal in SNpc dopaminergic neurons under neurodegenerative conditions that can cause PD.

It is likely that the beneficial effect of GDNF/Ret signaling might be further enhanced and more broadly applicable for the heterogeneity of idiopathic PD patients if combined with other neuroprotective and regenerative signals such as for example parkin overexpression. ${ }^{29,53}$ Further experiments are needed to support this hypothesis.

\section{Materials and Methods}

Recombinant vector production. Recombinant AAV5 viruses expressing GDNF and EGFP were produced as described. ${ }^{34}$

Animal procedures. Transgenic mice were kept on a C57BI/6J background and were previously described. ${ }^{23,30}$ All experimental animal procedures were conducted according to approved experimental animal licenses issued by the responsible animal welfare authority (Niedersächsisches Landesamt für Verbraucherschutz und Lebensmittelsicherheit) and controlled by the local animal welfare committee of the University Medical Center Göttingen. 
Unilateral stereotactical injection of $3 \times 10^{9}$ vector genomes $(\mathrm{vg})$ of AAV5- GDNF or AAV5-EGFP in 2-3-month-old male mice and MPTP treatment was done as previously described. ${ }^{34}$ Animals were either sacrificed by cervical dislocation, the striata dissected, frozen and stored at $-80^{\circ} \mathrm{C}$ or animals were sacrificed using $\mathrm{CO}_{2}$ and after transcardial perfusion (10 min $0.1 \mathrm{M} \mathrm{PBS}, \mathrm{pH} 7.4$ and $10 \mathrm{~min} 4 \%$ PFA in PBS) the whole brain was fixed and cryoprotected (24 h in 4\% PFA and $48 \mathrm{~h}$ in $30 \%$ sucrose at $4^{\circ} \mathrm{C}$ ).

Detection and quantification of GDNF. GDNF was quantified by IHC staining and ELISA as described. ${ }^{34}$

Histology. Stereological counting of dopaminergic neurons was performed by the optical fractionation method using Stereolnvestigator as described. ${ }^{30}$ Glia cell and dopaminergic fiber density quantification in the striatum was performed as described. 23,30

Primary antibodies were used in the following concentrations: anti-TH (mouse, $1: 2000$, DiaSorin), anti-DAT (rat, $1: 500$, Millipore), anti-GFAP (rabbit, $1: 300$, DacoCytomation), and anti-lba-1 (rabbit, $1: 500$, Wako).

Quantification of dopamine, DOPAC and HVA. Catecholamine levels were determined by ion-pair HPLC with electrochemical detection essentially as described. $^{30}$

Statistical analysis. Data are expressed as means \pm S.D. To compare different group means for independent samples, the statistical analysis was performed by ANOVA, followed by Tukey's post hoc test (GraphPad Prism 4.0). Significance levels were set at ${ }^{*} P<0.05,{ }^{* *} P<0.01$ and ${ }^{* *} P<0.001$.

\section{Conflict of Interest}

The authors declare no conflict of interest.

Acknowledgements. We thank Visitron Systems GmbH, Puchheim, Germany for improving the MetaMorph Macro for quantifying the striatal innervation, A Seeburg, IF Grimm and J Eisenmann for technical assistance and $\mathrm{K}$ Duncan and $P$ Nidadavolu for helpful comments on the manuscript. This work was supported by grants from the DFG (KR3529/4-1 to ERK) and the town of Hamburg (Lexi to ERK).

\section{Author contributions}

KT, DPM and ERK raised and genotyped the mice and analyzed the fiber density, gliosis and inflammation; AD stereotactically injected the mice and analyzed the cell number, striatal innervation and dopamine levels; JBS, SK and ERK designed the study; SK and ERK interpreted the data and wrote the manuscript with the help of JBS, AD, KT and DPM.

1. Airaksinen MS, Saarma M. The GDNF family: signalling, biological functions and therapeutic value. Nat Rev Neurosci 2002; 3: 383-394.

2. Paratcha G, Ledda F. GDNF and GFRalpha: a versatile molecular complex for developing neurons. Trends Neurosci 2008; 31: 384-391.

3. Manfredsson FP, Okun MS, Mandel RJ. Gene therapy for neurological disorders: challenges and future prospects for the use of growth factors for the treatment of Parkinson's disease. Curr Gene Ther 2009; 9: 375-388.

4. Aron L, Klein R. Repairing the parkinsonian brain with neurotrophic factors. Trends Neurosci 2011; 34: 88-100.

5. Kramer ER, Liss B. GDNF-Ret signaling in midbrain dopaminergic neurons and its implication for Parkinson disease. FEBS Lett 2015; 589: 3760-3772.

6. Wider C, Wszolek ZK. Movement disorders: insights into mechanisms and hopes for treatment. Lancet Neurol 2009; 8: 8-10.

7. Meissner WG, Frasier M, Gasser T, Goetz CG, Lozano A, Piccini $P$ et al. Priorities in Parkinson's disease research. Nat Rev Drug Discov 2011; 10: 377-393.

8. Goedert M, Spillantini MG, Del Tredici K, Braak H. 100 years of Lewy pathology. Nat Rev Neurol 2013; 9: 13-24.

9. Kirik D, Georgievska B, Bjorklund A. Localized striatal delivery of GDNF as a treatment for Parkinson disease. Nat Neurosci 2004; 7: 105-110.

10. Coune PG, Schneider BL, Aebischer P. Parkinson's disease: gene therapies. Cold Spring Harb Perspect Med 2012; 2: a009431.

11. Kordower JH, Bjorklund A. Trophic factor gene therapy for Parkinson's disease. Mov Disord 2013; 28: 96-109

12. Domanskyi A, Saarma M, Airavaara M. Prospects of neurotrophic factors for Parkinson's disease: comparison of protein and gene therapy. Hum Gene Ther 2015; 26: 550-559.
13. d'Anglemont de Tassigny X, Pascual A, Lopez-Barneo J. GDNF-based therapies, GDNF-producing interneurons, and trophic support of the dopaminergic nigrostriatal pathway. Implications for Parkinson's disease. Front Neuroanat 2015; 9: 10.

14. Chao CC, Ma YL, Chu KY, Lee EH. Integrin alphav and NCAM mediate the effects of GDNF on DA neuron survival, outgrowth, DA turnover and motor activity in rats. Neurobiol Aging 2003; 24: 105-116.

15. Paratcha $\mathrm{G}$, Ledda F, Ibanez CF. The neural cell adhesion molecule NCAM is an alternative signaling receptor for GDNF family ligands. Cell 2003; 113: 867-879.

16. Cao JP, Yu JK, Li C, Sun Y, Yuan HH, Wang HJ et al. Integrin beta1 is involved in the signaling of glial cell line-derived neurotrophic factor. J Comp Neurol 2008; 509: 203-210.

17. Bespalov MM, Sidorova YA, Tumova S, Ahonen-Bishopp A, Magalhaes AC, Kulesskiy E et al. Heparan sulfate proteoglycan syndecan-3 is a novel receptor for GDNF, neurturin, and artemin. J Cell Biol 2011; 192: 153-169.

18. Zuo T, Qin JY, Chen J, Shi Z, Liu M, Gao X et al. Involvement of N-cadherin in the protective effect of glial cell line-derived neurotrophic factor on dopaminergic neuron damage. Int J Mol Med 2013; 31: 561-568.

19. Schuchardt A, D'Agati V, Larsson-Blomberg L, Costantini F, Pachnis V. Defects in the kidney and enteric nervous system of mice lacking the tyrosine kinase receptor Ret. Nature 1994; 367: 380-383.

20. Marcos C, Pachnis V. The effect of the ret- mutation on the normal development of the central and parasympathetic nervous systems. Int J Dev Biol 1996; Suppl 1: 137S-138S.

21. Jain S, Naughton CK, Yang M, Strickland A, Vij K, Encinas M et al. Mice expressing a dominant-negative Ret mutation phenocopy human Hirschsprung disease and delineate a direct role of Ret in spermatogenesis. Development 2004; 131: 5503-5513.

22. Jain S, Golden JP, Wozniak D, Pehek E, Johnson EM Jr., Milbrandt J. RET is dispensable for maintenance of midbrain dopaminergic neurons in adult mice. J Neurosci 2006; 26: 11230-11238.

23. Kramer ER, Aron L, Ramakers GM, Seitz S, Zhuang X, Beyer K et al. Absence of Ret signaling in mice causes progressive and late degeneration of the nigrostriatal system. PLoS Biol 2007; 5: e39.

24. Aron L, Klein P, Pham TT, Kramer ER, Wurst W, Klein R. Pro-survival role for Parkinson's associated gene DJ-1 revealed in trophically impaired dopaminergic neurons. PLoS Biol 2010; 8: e1000349.

25. Meka DP, Muller-Rischart AK, Nidadavolu P, Mohammadi B, Motori E, Ponna SK et al. Parkin cooperates with GDNF/RET signaling to prevent dopaminergic neuron degeneration. $J$ Clin Invest 2015; 125: 1873-1885.

26. Pascual A, Hidalgo-Figueroa M, Piruat Jl, Pintado CO, Gomez-Diaz R, Lopez-Barneo J. Absolute requirement of GDNF for adult catecholaminergic neuron survival. Nat Neurosci 2008; 11: 755-761.

27. Kopra J, Vilenius C, Grealish S, Harma MA, Varendi K, Lindholm J et al GDNF is not required for catecholaminergic neuron survival in vivo. Nat Neurosci 2015; 18: 319-322.

28. Pascual A, Lopez-Barneo J. Reply to "GDNF is not required for catecholaminergic neuron survival in vivo". Nat Neurosci 2015; 18: 322-323.

29. Kramer ER. The neuroprotective and regenerative potential of parkin and GDNF/Ret signaling in the midbrain dopaminergic system. Neural Regen Res 2015; 10: 1752-1753.

30. Kowsky S, Poppelmeyer C, Kramer ER, Falkenburger BH, Kruse A, Klein R et al. RET signaling does not modulate MPTP toxicity but is required for regeneration of dopaminergic axon terminals. Proc Natl Acad Sci USA 2007; 104: 20049-20054.

31. Hidalgo-Figueroa M, Bonilla S, Gutierrez F, Pascual A, Lopez-Barneo J. GDNF is predominantly expressed in the $\mathrm{PV}+$ neostriatal interneuronal ensemble in normal mouse and after injury of the nigrostriatal pathway. J Neurosci 2012; 32: 864-872.

32. Kramer ER, Knott L, Su F, Dessaud E, Krull CE, Helmbacher F et al. Cooperation between GDNF/Ret and ephrinA/EphA4 signals for motor-axon pathway selection in the limb. Neuron 2006; 50: 35-47.

33. Zhuang X, Masson J, Gingrich JA, Rayport S, Hen R. Targeted gene expression in dopamine and serotonin neurons of the mouse brain. $J$ Neurosci Methods 2005; 143: 27-32.

34. Drinkut A, Tereshchenko Y, Schulz JB, Bahr M, Kugler S. Efficient gene therapy for Parkinson's disease using astrocytes as hosts for localized neurotrophic factor delivery. Mol Ther 2012; 20: 534-543.

35. Terzioglu M, Galter D. Parkinson's disease: genetic versus toxin-induced rodent models. FEBS J 2008; 275: 1384-1391.

36. Blesa J, Phani S, Jackson-Lewis V, Przedborski S. Classic and new animal models of Parkinson's disease. J Biomed Biotechnol 2012; 2012: 845618.

37. Klein RD, Sherman D, Ho WH, Stone D, Bennett GL, Moffat B et al. A GPI-linked protein that interacts with Ret to form a candidate neurturin receptor. Nature 1997; 387: 717-721.

38. Widenfalk J, Nosrat C, Tomac A, Westphal H, Hoffer B, Olson L. Neurturin and glial cell linederived neurotrophic factor receptor-beta (GDNFR-beta), novel proteins related to GDNF and GDNFR-alpha with specific cellular patterns of expression suggesting roles in the developing and adult nervous system and in peripheral organs. J Neurosci 1997; 17: 8506-8519.

39. Horger BA, Nishimura MC, Armanini MP, Wang LC, Poulsen KT, Rosenblad C et al. Neurturin exerts potent actions on survival and function of midbrain dopaminergic neurons. J Neurosci 1998; 18: 4929-4937. 
40. Gonzalez-Reyes LE, Verbitsky M, Blesa J, Jackson-Lewis V, Paredes D, Tillack K et al. Sonic hedgehog maintains cellular and neurochemical homeostasis in the adult nigrostriatal circuit. Neuron 2012; 75: 306-319.

41. Migliore MM, Ortiz R, Dye S, Campbell RB, Amiji MM, Waszczak BL. Neurotrophic and neuroprotective efficacy of intranasal GDNF in a rat model of Parkinson's disease. Neuroscience 2014; 274: 11-23.

42. Hewitt FC, Li C, Gray SJ, Cockrell S, Washburn M, Samulski RJ. Reducing the risk of adenoassociated virus (AAV) vector mobilization with AAV type 5 vectors. J Virol 2009; 83: 3919-3929.

43. Do Thi NA, Saillour P, Ferrero L, Dedieu JF, Mallet J, Paunio T. Delivery of GDNF by an E1, E3/E4 deleted adenoviral vector and driven by a GFAP promoter prevents dopaminergic neuron degeneration in a rat model of Parkinson's disease. Gene Ther 2004; 11: 746-756.

44. Ericson C, Georgievska B, Lundberg C. Ex vivo gene delivery of GDNF using primary astrocytes transduced with a lentiviral vector provides neuroprotection in a rat model of Parkinson's disease. Eur J Neurosci 2005; 22: 2755-2764.

45. Georgievska B, Kirik D, Bjorklund A. Aberrant sprouting and downregulation of tyrosine hydroxylase in lesioned nigrostriatal dopamine neurons induced by long-lasting overexpression of glial cell line derived neurotrophic factor in the striatum by lentiviral gene transfer. Exp Neurol 2002; 177: 461-474.

46. Georgievska B, Kirik D, Bjorklund A. Overexpression of glial cell line-derived neurotrophic factor using a lentiviral vector induces time- and dose-dependent downregulation of tyrosine hydroxylase in the intact nigrostriatal dopamine system. J Neurosci 2004; 24: 6437-6445.

47. Sajadi A, Bauer M, Thony B, Aebischer P. Long-term glial cell line-derived neurotrophic factor overexpression in the intact nigrostriatal system in rats leads to a decrease of dopamine and increase of tetrahydrobiopterin production. I Neurochem 2005; 93: 1482-1486.

48. Do Thi NA, Saillour P, Ferrero L, Paunio T, Mallet J. Does neuronal expression of GDNF effectively protect dopaminergic neurons in a rat model of Parkinson's disease? Gene Ther 2007; 14: 441-450.

49. Lindgren N, Francardo V, Quintino L, Lundberg C, Cenci MA. A model of GDNF gene therapy in mice with 6-Hydroxydopamine lesions: time course of Neurorestorative effects and ERK1/2 activation. J Parkinson's Dis 2012; 2: 333-348.

50. Lindgren N, Leak RK, Carlson KM, Smith AD, Zigmond MJ. Activation of the extracellular signal-regulated kinases 1 and 2 by glial cell line-derived neurotrophic factor and its relation to neuroprotection in a mouse model of Parkinson's disease. J Neurosci Res 2008; 86: 2039-2049.
51. Du $Y$, Li X, Yang D, Zhang $X$, Chen S, Huang $\mathrm{K}$ et al. Multiple molecular pathways are involved in the neuroprotection of GDNF against proteasome inhibitor induced dopamine neuron degeneration in vivo. Exp Biol Med (Maywood) 2008; 233: 881-890.

52. Klein P, Muller-Rischart AK, Motori E, Schonbauer C, Schnorrer F, Winklhofer KF et al. Ret rescues mitochondrial morphology and muscle degeneration of Drosophila Pink1 mutants. EMBO J 2014; 33: 341-355.

53. Kramer ER. Crosstalk of parkin and Ret in dopaminergic neurons. Oncotarget 2015; 6: 15704-15705.

54. Bespalov MM, Saarma M. GDNF family receptor complexes are emerging drug targets. Trends Pharmacol Sci 2007; 28: 68-74.

55. Tokugawa K, Yamamoto K, Nishiguchi M, Sekine T, Sakai M, Ueki T et al. XIB4035, a novel nonpeptidyl small molecule agonist for GFRalpha-1. Neurochem Int 2003; 42: 81-86.

56. Decressac M, Kadkhodaei B, Mattsson B, Laguna A, Perlmann T, Bjorklund A. alphaSynuclein-induced down-regulation of Nurr1 disrupts GDNF signaling in nigral dopamine neurons. Sci Transl Med 2012; 4: 163ra156.

57. Farrer MJ. Genetics of Parkinson disease: paradigm shifts and future prospects. Nat Rev Genet 2006; 7: 306-318.

58. Hoffer BJ, Harvey BK. Is GDNF beneficial in Parkinson disease? Nat Rev Neurol 2011; 7: 600-602.

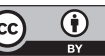

Cell Death and Disease is an open-access journal published by Nature Publishing Group. This work is licensed under a Creative Commons Attribution 4.0 International License. The images or other third party material in this article are included in the article's Creative Commons license, unless indicated otherwise in the credit line; if the material is not included under the Creative Commons license, users will need to obtain permission from the license holder to reproduce the material. To view a copy of this license, visit http://creativecommons.org/licenses/by/4.0/

(C) The Author(s) 2016

Supplementary Information accompanies this paper on Cell Death and Disease website (http://www.nature.com/cddis) 